International Journal of Bifurcation and Chaos

(c) World Scientific Publishing Company

\title{
Pullback attractors for differential equations with multiple variable delays in Lipschitz non-linearities
}

\author{
TOMÁS CARABALLO \\ Dpto. Ecuaciones Diferenciales y Análisis Numérico, Facultad de Matemáticas \\ Universidad de Sevilla, Campus Reina Mercedes, Apdo. de Correos 116041080 Sevilla, Spain. \\ caraball@us.es \\ GÁBOR KISS \\ Department of Mathematics \\ University of Durham \\ Durham \\ United Kingdom DH1 3LE \\ gabor.kiss@durham.ac.uk \\ TAKESHI TANIGUCHI \\ Division of Mathematical Sciences, Graduate School of Comparative Culture \\ Kurume University, Miimachi, Kurume \\ Fukuoka 839-8502, Japan \\ takeshi_taniguchi@kurume-u.ac.jp \\ Received (to be inserted by publisher)
}

\begin{abstract}
We establish results on the existence of pullback attractors for non-autonomous functional differential equations with multiple delays appearing within non-linear Lipschitz terms. The results are complementary to recently presented findings in [Caraballo \& Kiss, 2013b,a], and they extend the class of non-linearities to which existence results can be established by improving on a condition presented in [Caraballo \& Kiss, 2013b].
\end{abstract}

Keywords: Delay differential equation, non-autonomous dynamics, pullback attractors

\section{Introduction}

Our knowledge on equations with time-varying delays is relatively patchy; this fact is also mentioned in [Mallet-Paret \& Nussbaum, 2013] where the analyticity of solutions of equations of this type is investigated. The objective of the present work is to contribute to the analysis of the long-time behaviour of differential equations of the form

$$
\frac{d}{d t} D\left(t, x_{t}\right)=F_{0}(t, x(t))+\sum_{i=1}^{m} F_{i}\left(x\left(t-\rho_{i}(t)\right)\right),
$$

mainly focusing on the existence of pullback attractors. Throughout the paper, for $r>0, C=C\left([-r, 0] ; \mathbb{R}^{n}\right)$ denotes the Banach space of continuous functions with the sup-norm. We assume that the difference operator $D: \mathbb{R} \times C \rightarrow \mathbb{R}^{n}$ is continuous together with its first and second Fréchet derivatives with respect 
to its second variable; and the first and the second derivatives of $D$ with respect to the second variable are continuous at zero. In this paper, we consider two particular family of difference operators. For the delay terms, we assume that $\rho_{i}: \mathbb{R} \rightarrow[0, r]$ is a measurable function, and $r$ is a finite number. Lastly, our standing assumption will be the following:

(A1) For $i=1, \ldots, m$, there exists $L_{i}>0$, such that for any $x, y \in \mathbb{R}^{n}$

$$
\left|F_{i}(x)-F_{i}(y)\right| \leq L_{i}|x-y|
$$

and $F_{i}(0)=0$.

Under these and some difference-operator specific assumptions on $F_{0}$, we generalise very recent results presented in [Caraballo \& Kiss, 2013b] and [Caraballo \& Kiss, 2013a] which respectively deals with the existence of attractors for delay differential equations and neutral differential equations with time varying delays.

In the proof of our results we will use the following technical result proved as Lemma 3.1 in [Chen, 2010] and which is formulated as follows.

Lemma 1. Let $y:\left[t_{0}-t, \infty\right) \rightarrow \mathbb{R}^{+}$be a function such that for some $\gamma>0$ there are constants $\kappa, \kappa^{\prime}>0$ $\frac{\kappa^{\prime}}{\gamma}<1$ and

$$
y(t) \leq\left\{\begin{array}{l}
\kappa e^{-\gamma t}+\kappa^{\prime} \int_{t_{0}}^{t} e^{-\gamma(t-s)} \sup _{\theta \in[-r, 0]} y(s+\theta) d s, t>t_{0} \\
\kappa e^{-\gamma t}, t \in\left[t_{0}-r, t_{0}\right] .
\end{array}\right.
$$

Then

$$
y(t) \leq \kappa e^{-\mu t}, t \geq t_{0}-r
$$

where $\mu$ is the positive solution of $\frac{\kappa^{\prime}}{\gamma-\mu} e^{\mu r}=1$.

\section{Processes}

It is known that (see [Hale \& Verduyn Lunel, 1993]) if an initial function $\phi \in C$ is prescribed at the initial time $s \in \mathbb{R}$, the basic theory of delay differential equations implies the existence of the unique solution $x(\cdot ; s, \phi)$ of $(1)$ on $[s-r, \infty)$, which satisfies, in addition, the initial condition $x_{s}(\cdot)=\phi$, in other words, $x_{s}(\theta)=x(s+\theta)=\phi(\theta)$ for all $\theta \in[-r, 0]$. The unique solution of the initial value problems associated to (1) defines the solution map $U(t, s): C \ni \phi \mapsto x_{t}(\cdot ; s, \phi) \in C$ for $t \geq s$, which is, in fact, a process (also called a two-parameters semigroup), i.e.

- $U(t, s): C \rightarrow C$ is a continuous map for all $t \geq s$;

- $U(s, s)=i d_{C}$, the identity on $C$, for all $s \in \mathbb{R}$,

- $U(t, s)=U(t, \tau) U(\tau, s)$ for $t \geq \tau \geq s$.

As in the autonomous case, we look for invariant attracting sets. First, we introduce the Hausdorff semi-distance between subsets $A$ and $B$ in a metric space $(X, d)$ as

$$
\operatorname{dist}(A, B)=\sup _{a \in A} \inf _{b \in B} d(a, b) \text {. }
$$

Definition 2.1. Let $U$ be a process on a complete metric space $X$. A family of compact sets $\{\mathcal{A}(t)\}_{t \in \mathbb{R}}$ is said to be a (global) pullback attractor for $U$ if, for all $s \in \mathbb{R}$, it satisfies

- $U(t, s) \mathcal{A}(s)=\mathcal{A}(t)$ for all $t \geq s$, and

- $\lim _{s \rightarrow \infty} \operatorname{dist}(U(t, t-s) D, \mathcal{A}(t))=0$, for all bounded subsets $D$ of $X$.

Definition 2.2. $\{B(t)\}_{t \in \mathbb{R}}$ is said to be absorbing with respect to the process $U$ if, for $t \in \mathbb{R}$ and $D \subset X$ bounded, there exists $T_{D}(t)>0$ such that for all $\tau \geq T_{D}(t)$

$$
U(t, t-\tau) D \subset B(t) .
$$


The following result (see, e.g. [Schmalfuss, 1992]) shows that the existence of a family of compact absorbing sets implies the existence of a pullback attractor.

Theorem 1. Let $U(t, s)$ be a process on a complete metric space $X$. If there exists a family $\{B(t)\}_{t \in \mathbb{R}}$ of compact absorbing sets then, there exists a pullback attractor $\{\mathcal{A}(t)\}_{t \in \mathbb{R}}$ such that $\mathcal{A}(t) \subset B(t)$ for all $t \in \mathbb{R}$. Furthermore,

$$
\mathcal{A}(t)=\overline{\bigcup_{\substack{D \subset X \\ \text { bounded }}} \Lambda_{D}(t)}
$$

where

$$
\Lambda_{D}(t)=\bigcap_{n \in \mathbb{N}} \overline{\bigcup_{s \geq n} U(t, t-s) D}
$$

The next result will be crucial in our analysis.

Theorem 2. (see, e.g., Caraballo 8 Kiss [Caraballo 8 Kiss, 2013b]) Suppose that U(t,s) maps bounded sets into bounded sets and there exists a family $\{B(t)\}_{t \in \mathbb{R}}$ of bounded absorbing sets for $U$. Then there exists a pullback attractor for problem (1).

\section{Main results}

In this section we will establish the main results of our paper. First we will consider the case of retarded equations, and we will finally analyze some neutral delay equations.

\subsection{Retarded equations}

In this subsection we assume that $D \phi=\phi(0)$ for all $\phi \in C$, that is, (1) becomes a delay differential equation of the form

$$
\dot{x}(t)=F_{0}(t, x(t))+\sum_{i=1}^{m} F_{i}\left(x\left(t-\rho_{i}(t)\right)\right) .
$$

In addition to (A1), we assume

(A2) $F_{0}: \mathbb{R}^{n+1} \rightarrow \mathbb{R}^{n}$ is continuous and there exist $\alpha_{0}>0$, and a non-negative measurable function $\beta(\cdot)$ such that

$$
\left\langle F_{0}(t, x), x\right\rangle \leq-\alpha_{0}|x|^{2}+\beta(t), t \in \mathbb{R}, x \in \mathbb{R}^{n}
$$

In [Caraballo \& Kiss, 2013b] the following result was proved:

Theorem 3. (Caraballo and Kiss [Caraballo E3 Kiss, 2013b, Theorem 3.4]) Assume that hypotheses (A1) and (A2) are satisfied and that there exists $\lambda>\alpha_{0}$ such that

$$
\int_{-\infty}^{t} e^{\lambda s} \beta(s) d s<+\infty, \quad \forall t \in \mathbb{R}
$$

Then, if $\rho_{i}, i=1, \ldots, m$, is measurable and $L_{i}<\frac{\alpha_{0}}{m}$, there exists a family of bounded absorbing sets, $\{B(t)\}_{t \in \mathbb{R}}$, and consequently, there exists a pullback attractor for the process generated by (3).

Remark 3.1. Notice that the assumption $\lambda>\alpha_{0}$ in Theorem 3 can be replaced by: there exists $\lambda>0$ such that

$$
\int_{-\infty}^{t} e^{\lambda s} \beta(s) d s<+\infty, \quad \forall t \in \mathbb{R} .
$$

Indeed, it is easy to see that if (5) holds for certain $\lambda_{0}$, then it also holds true for any $\lambda>\lambda_{0}$, and then we can pick one value bigger than $\alpha_{0}$.

Consequently, a first improvement is that in Theorem 3 we can assume $\lambda>0$. 
However, in case that the constant $\lambda$ belongs to the interval $\left(0, \alpha_{0}\right)$, then it is possible to prove a complementary result by imposing a different smallness condition on the Lipschitz terms. This is established in the next theorem.

Theorem 4. Assume that hypotheses (A1) and (A2) are satisfied and that there exists $\lambda \in\left(0, \alpha_{0}\right)$ such that

$$
\int_{-\infty}^{t} e^{\lambda s} \beta(s) d s<+\infty, \quad \forall t \in \mathbb{R}
$$

Then, if $\rho_{i}, i=1, \ldots, m$, is measurable and $\sum_{i=1}^{m} L_{i}^{2}<\frac{\lambda \alpha_{0}}{m}$, there exists a family of bounded absorbing sets, $\{B(t)\}_{t \in \mathbb{R}}$, and consequently, there exists a pullback attractor for the process generated by (3).

Proof. Let us consider the number $\lambda>0$ from (6), pick $\varepsilon=\frac{\alpha_{0}}{m}$, and denote $x(\tau)=x\left(\tau ; t_{0}-t, \psi\right), \tau \in$ $\left[t_{0}-t, t_{0}\right]$, for any $\psi \in C$ such that $\|\psi\| \leq d$, and $t_{0} \in \mathbb{R}$. Then, applying the Young inequality in the delay terms below, taking into account that $\lambda<\alpha_{0}$,

$$
\begin{aligned}
\frac{d}{d \tau} e^{\lambda \tau}|x(\tau)|^{2}= & \lambda e^{\lambda \tau}|x(\tau)|^{2}+2 e^{\lambda \tau}\left\langle x(\tau), F_{0}(\tau, x(\tau))\right\rangle \\
& +2 e^{\lambda \tau} \sum_{i=1}^{m}\left\langle x(\tau), F_{i}\left(x\left(\tau-\rho_{i}(\tau)\right)\right)\right\rangle \\
\leq & \left(\lambda-2 \alpha_{0}\right) e^{\lambda \tau}|x(\tau)|^{2}+2 e^{\lambda \tau} \beta(\tau)+e^{\lambda \tau}|x(\tau)|^{2} m \varepsilon \\
& +e^{\lambda \tau} \varepsilon^{-1} \sum_{i=1}^{m}\left|F_{i}\left(x\left(\tau-\rho_{i}(\tau)\right)\right)\right|^{2} \\
\leq & \left(\lambda-\alpha_{0}\right) e^{\lambda \tau}|x(\tau)|^{2}+2 e^{\lambda \tau} \beta(\tau) \\
& +e^{\lambda \tau} \varepsilon^{-1} \sum_{i=1}^{m} L_{i}^{2}\left|x\left(\tau-\rho_{i}(\tau)\right)\right|^{2} \\
\leq & 2 e^{\lambda \tau} \beta(\tau)+e^{\lambda \tau} \varepsilon^{-1} \sum_{i=1}^{m} L_{i}^{2}\left|x\left(\tau-\rho_{i}(\tau)\right)\right|^{2} .
\end{aligned}
$$

Integration on the interval $\left[t_{0}-t, \tau\right]$ yields that

$$
\begin{aligned}
e^{\lambda \tau}|x(\tau)|^{2}-e^{\lambda\left(t_{0}-t\right)}\left|x\left(t_{0}-t\right)\right|^{2} & \leq 2 \int_{t_{0}-t}^{\tau} e^{\lambda s} \beta(s) d s \\
& +\varepsilon^{-1} \sum_{i=1}^{m} L_{i}^{2} \int_{t_{0}-t}^{\tau} e^{\lambda s}\left|x\left(s-\rho_{i}(s)\right)\right|^{2} d s
\end{aligned}
$$

The integrand in the third sum can be estimated as follows

$$
\int_{t_{0}-t}^{\tau} e^{\lambda s}\left|x\left(s-\rho_{i}(s)\right)\right|^{2} d s \leq \int_{t_{0}-t}^{\tau} e^{\lambda s} \sup _{\theta \in[-r, 0]}|x(s+\theta)|^{2} d s .
$$

We thus have

$$
\begin{aligned}
|x(\tau)|^{2} \leq & e^{\lambda\left(t_{0}-t-\tau\right)}\left|x\left(t_{0}-t\right)\right|^{2} \\
& +e^{-\lambda \tau}\left(2 \int_{t_{0}-t}^{\tau} e^{\lambda s} \beta(s) d s+\varepsilon^{-1} \sum_{i=1}^{m} L_{i}^{2} \int_{t_{0}-t}^{\tau} e^{\lambda s} \sup _{\theta \in[-r, 0]}|x(s+\theta)|^{2} d s\right) .
\end{aligned}
$$


Now, (7) reads as

$$
\begin{aligned}
|x(\tau)|^{2} \leq & \left(e^{\lambda\left(t_{0}-t\right)} d^{2}+2 \int_{-\infty}^{t_{0}} e^{\lambda s} \beta(s) d s\right) e^{-\lambda \tau} \\
& +\left(\varepsilon^{-1} \sum_{i=1}^{m} L_{i}^{2}\right) \int_{t_{0}-t}^{\tau} e^{-\lambda(\tau-s)} \sup _{\theta \in[-r, 0]}|x(s+\theta)|^{2} d s .
\end{aligned}
$$

Let $\kappa=e^{\lambda\left(t_{0}-t\right)} d^{2}+2 \int_{-\infty}^{t_{0}} e^{\lambda s} \beta(s) d s, \kappa^{\prime}=\varepsilon^{-1} \sum_{i=1}^{m} L_{i}^{2}$ and $\gamma=\lambda$. Then,

$$
\frac{\kappa^{\prime}}{\gamma}=\frac{\varepsilon^{-1} \sum_{i=1}^{m} L_{i}^{2}}{\lambda}<1
$$

Thus, Lemma 1 implies that

$$
|x(\tau)|^{2} \leq \kappa e^{-\mu \tau}=\left(e^{\lambda\left(t_{0}-t\right)} d^{2}+2 \int_{-\infty}^{t_{0}} e^{\lambda s} \beta(s) d s\right) e^{-\mu \tau} .
$$

where $\mu$ is the positive solution of

$$
\frac{\varepsilon^{-1} \sum_{i=1}^{m} L_{i}^{2}}{\lambda-\mu} e^{\mu r}=1
$$

This allows us to obtain

$$
\begin{aligned}
\sup _{\theta \in[-r, 0]}\left|x\left(t_{0}+\theta\right)\right|^{2} & \leq\left(d^{2} e^{(\lambda-\mu) t_{0}} e^{-\lambda t}+2 \int_{-\infty}^{t_{0}} e^{\lambda s} \beta(s) d s\right) e^{\mu r} \\
& \leq\left(1+2 \int_{-\infty}^{t_{0}} e^{\lambda s} \beta(s) d s\right) e^{\mu r}
\end{aligned}
$$

provided

$$
t \geq T_{D}=\lambda^{-1} \log \left(d^{2} e^{(\lambda-\mu) t_{0}}\right) .
$$

Consequently, the family of bounded sets $\{B(t)\}_{t \in \mathbb{R}}$ in $C\left([-r, 0] ; \mathbb{R}^{n}\right)$ given by $B(t):=B(0 ; R(t))$, for all $t \in \mathbb{R}$, where $B(0 ; R(t))$ denotes the ball centered at zero with radius $R(t)=\left(1+2 \int_{-\infty}^{t} e^{\lambda s} \beta(s) d s\right) e^{\mu r}$, is absorbing. Taking into account that the associated process maps bounded sets into bounded sets of $C\left([-r, 0] ; \mathbb{R}^{n}\right)$, the existence of the pullback attractor is ensured by Theorem 2 (see also Theorem 2.4 in [Caraballo \& Kiss, 2013b]).

\subsection{Neutral equations}

This section focuses on equations with difference operators of the form

$$
D \phi=\phi(0)-g(\phi(-\sigma))
$$

where function $g$ maps $\mathbb{R}^{n}$ into itself. In other words, here we consider the family of neutral functional differential equations of the form:

$$
\frac{d}{d t}[x(t)-g(x(t-\sigma))]=F_{0}(t, x(t))+\sum_{i=1}^{m} F_{i}\left(x\left(t-\rho_{i}(t)\right)\right),
$$

where $\sigma>0$ and finite, and without loss of generality, we assume $\sigma<r$. In this section, we assume that our non-delay term satisfies a non-autonomous dissipativity condition, i.e. we impose the following assumption on $F_{0}$ :

(A3) $F_{0}: \mathbb{R}^{n+1} \rightarrow \mathbb{R}^{n}$ is continuous and there exist $\alpha_{0}>0$, and a non-negative measurable function $\beta(\cdot)$ such that 


$$
\left\langle F_{0}(t, \phi(0)), \phi(0)-g(\phi(-\sigma))\right\rangle \leq-\alpha_{0}|\phi(0)-g(\phi(-\sigma))|^{2}+\beta(t), t \in \mathbb{R}, x \in \mathbb{R}^{n} .
$$

In addition, we assume that for any continuous function $x: \mathbb{R} \rightarrow \mathbb{R}^{n}$, we have

$\left(A_{g}\right)|g(x(\tau-\sigma))|^{2} \leq c^{2}|x(\tau)|^{2}+\delta(\tau), 0<c<1, \tau \in \mathbb{R}$ such that $\delta(\tau) \in\left[0, M_{\delta}\right]$ for some $M_{\delta} \in \mathbb{R}^{+}$.

In [Caraballo \& Kiss, 2013a], the following result is shown.

Theorem 5. Assume that conditions (A1) and (A3) are satisfied and that there exists $\lambda \in\left(0, \alpha_{0}\right)$ such that

$$
\int_{-\infty}^{t} e^{\lambda s} \beta(s) d s<+\infty, \quad \forall t \in \mathbb{R}
$$

Then, if $\rho_{i}, i=1, \ldots, m$, is measurable, and

$$
\lambda(1-c)^{2} \alpha_{0} \geq 2 m \sum_{i=1}^{m} L_{i}^{2},
$$

there exists a family of bounded absorbing sets, $\{B(t)\}_{t \in \mathbb{R}}$, and consequently, there exists a pullback attractor for the process generated by (10).

The following theorems improve on (12) and complement Theorem 5. Before formulating them, we include a lemma from [Caraballo et al., 2007] which will be useful in subsequent computations.

Lemma 2. Let $0<\xi<1$. Then

$$
|u|^{2} \leq \frac{1}{1-\xi}|u-v|^{2}+\frac{1}{\xi}|v|^{2}
$$

for any $u, v \in \mathbb{R}^{n}$.

Now we can establish our main result in this section.

Theorem 6. Assume that assumptions (A1) and (A3) are satisfied and that there exists $\lambda>\alpha_{0}$ such that

$$
\int_{-\infty}^{t} e^{\lambda s} \beta(s) d s<+\infty, \quad \forall t \in \mathbb{R}
$$

Assume that $\rho_{i}, i=1, \ldots, m$, is measurable and that the following conditions hold:

$$
\begin{gathered}
\lambda<\alpha_{0}\left\{\left(c+\frac{1}{1-c}\right)\left(\left[c+\frac{1}{1-c}\right]-(1-c)^{2}\right)^{-1}\right\}, \\
\sum_{i=1}^{m} L_{i}^{2}<\frac{\alpha_{0}}{m}\left\{\lambda\left[(1-c)^{2}-\left[c+\frac{1}{1-c}\right]\right]+\left(c+\frac{1}{1-c}\right) \alpha_{0}\right\} .
\end{gathered}
$$

Then, there exists a family of bounded absorbing sets, $\{B(t)\}_{t \in \mathbb{R}}$, and consequently, there exists a pullback attractor for the process generated by (10).

Remark 3.2. It is assumed in [Caraballo \& Kiss, 2013b] that $\lambda \in\left(0, \alpha_{0}\right)$. Thus, in that sense, Theorem 6 is complementary to Theorem 5 .

Proof. Let us consider the number $\lambda>0$ from (13), pick $\varepsilon=\frac{\alpha_{0}}{m}$, and denote $x(\tau)=x\left(\tau ; t_{0}-t, \psi\right), \tau \in$ $\left[t_{0}-t, t_{0}\right]$, for any $\psi \in C$ such that $\|\psi\| \leq d$, and $t_{0} \in \mathbb{R}$. Then, applying again the Young inequality in the 
delay terms below, we get

$$
\begin{aligned}
\frac{d}{d \tau} e^{\lambda \tau}|x(\tau)-g(x(\tau-\sigma))|^{2}= & \lambda e^{\lambda \tau}|x(\tau)-g(x(\tau-\sigma))|^{2} \\
& +2 e^{\lambda \tau}\left\langle x(\tau)-g(x(\tau-\sigma)), F_{0}(\tau, x(\tau))\right\rangle \\
& +2 e^{\lambda \tau} \sum_{i=1}^{m}\left\langle x(\tau)-g(x(\tau-\sigma)), F_{i}\left(x\left(\tau-\rho_{i}(\tau)\right)\right)\right\rangle \\
\leq & \left(\lambda-2 \alpha_{0}\right) e^{\lambda \tau}|x(\tau)-g(x(\tau-\sigma))|^{2} \\
& +2 e^{\lambda \tau} \beta(\tau)+e^{\lambda \tau}|x(\tau)-g(x(\tau-\sigma))|^{2} m \varepsilon \\
& +e^{\lambda \tau} \varepsilon^{-1} \sum_{i=1}^{m}\left|F_{i}\left(x\left(\tau-\rho_{i}(\tau)\right)\right)\right|^{2} \\
\leq & \left(\lambda-\alpha_{0}\right) e^{\lambda \tau}|x(\tau)-g(x(\tau-\sigma))|^{2}+2 e^{\lambda \tau} \beta(\tau) \\
& +e^{\lambda \tau} \varepsilon^{-1} \sum_{i=1}^{m} L_{i}^{2}\left|x\left(\tau-\rho_{i}(\tau)\right)\right|^{2} \\
\leq & \left(\lambda-\alpha_{0}\right)\left(c+\frac{1}{1-c}\right) e^{\lambda \tau}|x(\tau)|^{2}+2 e^{\lambda \tau} \beta(\tau) \\
& +\frac{\left(\lambda-\alpha_{0}\right) M_{\delta} e^{\lambda \tau}+e^{\lambda \tau} \varepsilon^{-1} \sum_{i=1}^{m} L_{i}^{2}\left|x\left(\tau-\rho_{i}(\tau)\right)\right|^{2}}{c}
\end{aligned}
$$

Integration on the interval $\left[t_{0}-t, \tau\right]$ yields that

$$
\begin{aligned}
e^{\lambda \tau}|x(\tau)-g(x(\tau-\sigma))|^{2}- & e^{\lambda\left(t_{0}-t\right)}\left|x\left(t_{0}-t\right)-g\left(x\left(t_{0}-t-\sigma\right)\right)\right|^{2} \\
\leq & \left(\lambda-\alpha_{0}\right)\left(c+\frac{1}{1-c}\right) \int_{t_{0}-t}^{\tau} e^{\lambda s}|x(s)|^{2} d s \\
& +2 \int_{t_{0}-t}^{\tau} e^{\lambda s} \beta(s) d s+\frac{\left(\lambda-\alpha_{0}\right) M_{\delta}}{\lambda c} e^{\lambda \tau} \\
& +\varepsilon^{-1} \sum_{i=1}^{m} L_{i}^{2} \int_{t_{0}-t}^{\tau} e^{\lambda s}\left|x\left(s-\rho_{i}(s)\right)\right|^{2} d s
\end{aligned}
$$

The integrand in the third sum can be estimated

$$
\int_{t_{0}-t}^{\tau} e^{\lambda s}\left|x\left(s-\rho_{i}(s)\right)\right|^{2} d s \leq \int_{t_{0}-t}^{\tau} e^{\lambda s} \sup _{\theta \in[-r, 0]}|x(s+\theta)|^{2} d s
$$

Thus we have

$$
\begin{aligned}
|x(\tau)-g(x(\tau-\sigma))|^{2} \leq & e^{\lambda\left(t_{0}-t-\tau\right)}\left(\frac{\left|x\left(t_{0}-t\right)\right|^{2}}{1-c}+c\left|x\left(t_{0}-t\right)\right|^{2}+\frac{M_{\delta}}{c}\right) \\
& +\left(\lambda-\alpha_{0}\right)\left(c+\frac{1}{1-c}\right) \int_{t_{0}-t}^{\tau} e^{\lambda(s-\tau)}|x(s)|^{2} d s+\frac{\left(\lambda-\alpha_{0}\right) M_{\delta}}{\lambda c} \\
& +e^{-\lambda \tau}\left(2 \int_{t_{0}-t}^{\tau} e^{\lambda s} \beta(s) d s+\varepsilon^{-1} \sum_{i=1}^{m} L_{i}^{2} \int_{t_{0}-t}^{\tau} e^{\lambda s} \sup _{\theta \in[-r, 0]}|x(s+\theta)|^{2} d s\right) .
\end{aligned}
$$


Now, we obtain

$$
\begin{aligned}
|x(\tau)|^{2} \leq \frac{1}{1-} & \left\{\left(e^{\lambda\left(t_{0}-t\right)}\left(\frac{d^{2}}{1-c}+d^{2} c+\frac{M_{\delta}}{c}\right)\right.\right. \\
& \left.+2 \int_{-\infty}^{t_{0}} e^{\lambda s} \beta(s) d s+\frac{\left(\lambda-\alpha_{0}\right) M_{\delta} e^{\lambda t_{0}}}{\lambda c}\right) e^{-\lambda \tau} \\
& \left.+\left(\left(\lambda-\alpha_{0}\right)\left(c+\frac{1}{1-c}\right)+\varepsilon^{-1} \sum_{i=1}^{m} L_{i}^{2}\right) \int_{t_{0}-t}^{\tau} e^{-\lambda(\tau-s)} \sup _{\theta \in[-r, 0]}|x(s+\theta)|^{2} d s\right\} \\
+ & \left(c|x(\tau)|^{2}+\frac{M_{\delta}}{c}\right) .
\end{aligned}
$$

And so,

$$
\begin{aligned}
|x(\tau)|^{2} \leq & \frac{1}{(1-c)^{2}}\left\{\left(e^{\lambda\left(t_{0}-t\right)}\left(\frac{d^{2}}{1-c}+d^{2} c+\frac{M_{\delta}}{c}\right)\right.\right. \\
& \left.+2 \int_{-\infty}^{t_{0}} e^{\lambda s} \beta(s) d s+\frac{\left(\lambda-\alpha_{0}\right) M_{\delta} e^{\lambda t_{0}}}{\lambda c}+\frac{(1-c) M_{\delta}}{c} e^{\lambda \tau}\right) e^{-\lambda \tau} \\
& \left.+\left(\left(\lambda-\alpha_{0}\right)\left(c+\frac{1}{1-c}\right)+\varepsilon^{-1} \sum_{i=1}^{m} L_{i}^{2}\right) \int_{t_{0}-t}^{\tau} e^{-\lambda(\tau-s)} \sup _{\theta \in[-r, 0]}|x(s+\theta)|^{2} d s\right\} .
\end{aligned}
$$

Let

$$
\begin{gathered}
\kappa=\frac{e^{\lambda\left(t_{0}-t\right)}\left(\frac{d^{2}}{1-c}+d^{2} c+\frac{M_{\delta}}{c}\right)+2 \int_{-\infty}^{t_{0}} e^{\lambda s} \beta(s) d s+\frac{\left(\lambda-\alpha_{0}\right) M_{\delta} e^{\lambda t_{0}}}{\lambda c}+\frac{(1-c) M_{\delta}}{c} e^{\lambda \tau}}{(1-c)^{2}}, \\
\kappa^{\prime}=\frac{\left(\lambda-\alpha_{0}\right)\left(c+\frac{1}{1-c}\right)+\varepsilon^{-1} \sum_{i=1}^{m} L_{i}^{2}}{(1-c)^{2}}
\end{gathered}
$$

and $\gamma=\lambda$.

Then, using (14) and (15), it follows that

$$
\begin{aligned}
\frac{\kappa^{\prime}}{\gamma} & =\frac{\left(\lambda-\alpha_{0}\right)\left(c+\frac{1}{1-c}\right)+\varepsilon^{-1} \sum_{i=1}^{m} L_{i}^{2}}{\lambda(1-c)^{2}} \\
& <\frac{\left(\lambda-\alpha_{0}\right)\left(c+\frac{1}{1-c}\right)+\lambda\left((1-c)^{2}-\left(c+\frac{1}{1-c}\right)\right)+\left(c+\frac{1}{1-c}\right) \alpha_{0}}{\lambda(1-c)^{2}} \\
& =1 .
\end{aligned}
$$

Thus, Lemma 1 implies that

$$
\begin{aligned}
|x(\tau)|^{2} & \leq \kappa e^{-\mu \tau} \\
& =\frac{\left(e^{\lambda\left(t_{0}-t\right)}\left(\frac{d^{2}}{1-c}+d^{2} c+\frac{M_{\delta}}{c}\right)+2 \int_{-\infty}^{t_{0}} e^{\lambda s} \beta(s) d s+\frac{\left(\lambda-\alpha_{0}\right) M_{\delta} e^{\lambda t_{0}}}{\lambda c}+\frac{(1-c) M_{\delta}}{c} e^{\lambda \tau}\right)}{(1-c)^{2}} e^{-\mu \tau},
\end{aligned}
$$

where $\mu$ is the positive solution of

$$
\frac{\kappa^{\prime}}{\lambda-\mu} e^{\mu r}=1
$$


Thus we obtain that

$$
\begin{aligned}
& \sup _{\theta \in[-r, 0]}\left|x\left(t_{0}+\theta\right)\right|^{2} \\
& \leq \frac{\left(\left(\frac{d^{2}}{1-c}+d^{2} c+\frac{M_{\delta}}{c}\right) e^{(\lambda-\mu) t_{0}} e^{-\lambda t}+2 \int_{-\infty}^{t_{0}} e^{\lambda s} \beta(s) d s+\frac{\left(\lambda-\alpha_{0}\right) M_{\delta} e^{\lambda t_{0}}}{\lambda c}+\frac{(1-c) M_{\delta}}{c} e^{\lambda \tau}\right)}{(1-c)^{2}} e^{\mu r} \\
& \quad \leq \frac{\left(1+2 \int_{-\infty}^{t_{0}} e^{\lambda s} \beta(s) d s+\frac{\left(\lambda-\alpha_{0}\right) M_{\delta} e^{\lambda t_{0}}}{\lambda c}+\frac{(1-c) M_{\delta}}{c} e^{\lambda \tau}\right)}{(1-c)^{2}} e^{\mu r}
\end{aligned}
$$

provided

$$
t \geq T_{D}=\lambda^{-1} \log \left(\left(\frac{d^{2}}{1-c}+d^{2} c+\frac{M_{\delta}}{c}\right) e^{(\lambda-\mu) t_{0}}\right)
$$

Consequently, the family of bounded sets $\{B(t)\}_{t \in \mathbb{R}}$ in $C\left([-r, 0] ; \mathbb{R}^{n}\right)$ given by $B(t):=B(0 ; R(t))$, for all $t \in \mathbb{R}$, where $B(0 ; R(t))$ denotes the ball centred at zero with radius $R(t)=$ $\frac{\left(1+2 \int_{-\infty}^{t} e^{\lambda s} \beta(s) d s+\frac{\left(\lambda-\alpha_{0}\right) M_{\delta} e^{\lambda t}}{\lambda c}+\frac{(1-c) M_{\delta}}{c} e^{\lambda \tau}\right)}{(1-c)^{2}} e^{\mu r}$, is absorbing. Taking into account again that the associated process maps bounded sets into bounded sets of $C\left([-r, 0] ; \mathbb{R}^{n}\right)$, the existence of the pullback attractor is ensured again by Theorem 2 .

The next result improves on condition (12).

Theorem 7. Assume that hypotheses (A1) and (A3) are satisfied and that there exists $\lambda \in\left(0, \alpha_{0}\right)$ such that

$$
\int_{-\infty}^{t} e^{\lambda s} \beta(s) d s<+\infty, \quad \forall t \in \mathbb{R}
$$

Then, if $\rho_{i}, i=1, \ldots, m$, is measurable, and

$$
\sum_{i=1}^{m} L_{i}^{2}<\frac{\lambda(1-c)^{2} \alpha_{0}}{m}
$$

there exists a family of bounded absorbing sets, $\{B(t)\}_{t \in \mathbb{R}}$, and consequently, there exists a pullback attractor for the process generated by (10).

Proof. Let us consider the number $\lambda \in\left(0, \alpha_{0}\right)$ from (20), pick $\varepsilon=\frac{\alpha_{0}}{m}$, and denote $x(\tau)=x\left(\tau ; t_{0}-t, \psi\right), \tau \in$ $\left[t_{0}-t, t_{0}\right]$, for any $\psi \in C$ such that $\|\psi\| \leq d$, and $t_{0} \in \mathbb{R}$. Then, applying once again the Young inequality 
in the delay terms below, we obtain

$$
\begin{aligned}
\frac{d}{d \tau} e^{\lambda \tau}|x(\tau)-g(x(\tau-\sigma))|^{2}= & \lambda e^{\lambda \tau}|x(\tau)-g(x(\tau-\sigma))|^{2} \\
& +2 e^{\lambda \tau}\left\langle x(\tau)-g(x(\tau-\sigma)), F_{0}(\tau, x(\tau))\right\rangle \\
& +2 e^{\lambda \tau} \sum_{i=1}^{m}\left\langle x(\tau)-g(x(\tau-\sigma)), F_{i}\left(x\left(\tau-\rho_{i}(\tau)\right)\right)\right\rangle \\
\leq & \left(\lambda-2 \alpha_{0}\right) e^{\lambda \tau}|x(\tau)-g(x(\tau-\sigma))|^{2} \\
& +2 e^{\lambda \tau} \beta(\tau)+e^{\lambda \tau}|x(\tau)-g(x(\tau-\sigma))|^{2} m \varepsilon \\
& +e^{\lambda \tau} \varepsilon^{-1} \sum_{i=1}^{m}\left|F_{i}\left(x\left(\tau-\rho_{i}(\tau)\right)\right)\right|^{2} \\
\leq & \left(\lambda-\alpha_{0}\right) e^{\lambda \tau}|x(\tau)-g(x(\tau-\sigma))|^{2}+2 e^{\lambda \tau} \beta(\tau) \\
& +e^{\lambda \tau} \varepsilon^{-1} \sum_{i=1}^{m} L_{i}^{2}\left|x\left(\tau-\rho_{i}(\tau)\right)\right|^{2} \\
\leq & 2 e^{\lambda \tau} \beta(\tau)+e^{\lambda \tau} \varepsilon^{-1} \sum_{i=1}^{m} L_{i}^{2}\left|x\left(\tau-\rho_{i}(\tau)\right)\right|^{2} .
\end{aligned}
$$

Integration on the interval $\left[t_{0}-t, \tau\right]$ yields that

$$
\begin{array}{r}
e^{\lambda \tau}|x(\tau)-g(x(\tau-\sigma))|^{2}-e^{\lambda\left(t_{0}-t\right)}\left|x\left(t_{0}-t\right)-g\left(x\left(t_{0}-t-\sigma\right)\right)\right|^{2} \\
\leq 2 \int_{t_{0}-t}^{\tau} e^{\lambda s} \beta(s) d s+\varepsilon^{-1} \sum_{i=1}^{m} L_{i}^{2} \int_{t_{0}-t}^{\tau} e^{\lambda s}\left|x\left(s-\rho_{i}(s)\right)\right|^{2} d s
\end{array}
$$

The integrand in the last sum can be estimated as

$$
\int_{t_{0}-t}^{\tau} e^{\lambda s}\left|x\left(s-\rho_{i}(s)\right)\right|^{2} d s \leq \int_{t_{0}-t}^{\tau} e^{\lambda s} \sup _{\theta \in[-r, 0]}|x(s+\theta)|^{2} d s .
$$

And we then have

$$
\begin{aligned}
& |x(\tau)-g(x(\tau-\sigma))|^{2} \\
& \leq e^{\lambda\left(t_{0}-t-\tau\right)}\left(\frac{\left|x\left(t_{0}-t\right)\right|^{2}}{1-c}+c\left|x\left(t_{0}-t\right)\right|^{2}+\frac{M_{\delta}}{c}\right) \\
& \quad+e^{-\lambda \tau}\left(2 \int_{t_{0}-t}^{\tau} e^{\lambda s} \beta(s) d s+\varepsilon^{-1} \sum_{i=1}^{m} L_{i}^{2} \int_{t_{0}-t}^{\tau} e^{\lambda s} \sup _{\theta \in[-r, 0]}|x(s+\theta)|^{2} d s\right) .
\end{aligned}
$$

Now, using again Lemma 2, we deduce

$$
\begin{aligned}
|x(\tau)|^{2} \leq & \frac{1}{1-c}|x(\tau)-g(x(\tau-\sigma))|^{2}+\frac{1}{c}|g(x(\tau-\sigma))|^{2} \\
\leq & \frac{1}{1-c}\left(e^{\lambda\left(t_{0}-t\right)}\left(\frac{d^{2}}{1-c}+d^{2} c+\frac{M_{\delta}}{c}\right)+2 \int_{-\infty}^{t_{0}} e^{\lambda s} \beta(s) d s\right) e^{-\lambda \tau} \\
& +((1-c) \varepsilon)^{-1} \sum_{i=1}^{m} L_{i}^{2} \int_{t_{0}-t}^{\tau} e^{-\lambda(\tau-s)} \sup _{\theta \in[-r, 0]}|x(s+\theta)|^{2} d s \\
& +c|x(\tau)|^{2}+\frac{M_{\delta}}{c}
\end{aligned}
$$


Consequently

$$
\begin{aligned}
|x(\tau)|^{2} \leq & \frac{1}{(1-c)^{2}}|x(\tau)-g(x(\tau-\sigma))|^{2}+\frac{1}{c(1-c)}|g(x(\tau-\sigma))|^{2} \\
\leq & \frac{1}{(1-c)^{2}}\left(e^{\lambda\left(t_{0}-t\right)}\left(\frac{d^{2}}{1-c}+d^{2} c+\frac{M_{\delta}}{c}\right)+2 \int_{-\infty}^{t_{0}} e^{\lambda s} \beta(s) d s\right) e^{-\lambda \tau} \\
& +\left((1-c)^{2} \varepsilon\right)^{-1} \sum_{i=1}^{m} L_{i}^{2} \int_{t_{0}-t}^{\tau} e^{-\lambda(\tau-s)} \sup _{\theta \in[-r, 0]}|x(s+\theta)|^{2} d s \\
& +\frac{M_{\delta}}{c(1-c)} \\
\leq & \frac{1}{(1-c)^{2}}\left(e^{\lambda\left(t_{0}-t\right)}\left(\frac{d^{2}}{1-c}+d^{2} c+\frac{M_{\delta}}{c}\right)+2 \int_{-\infty}^{t_{0}} e^{\lambda s} \beta(s) d s\right) e^{-\lambda \tau} \\
& +\left((1-c)^{2} \varepsilon\right)^{-1} \sum_{i=1}^{m} L_{i}^{2} \int_{t_{0}-t}^{\tau} e^{-\lambda(\tau-s)} \sup _{\theta \in[-r, 0]}|x(s+\theta)|^{2} d s \\
& +\frac{M_{\delta} e^{\lambda t_{0}}}{c(1-c)} e^{-\lambda \tau} .
\end{aligned}
$$

Let

$$
\begin{gathered}
\kappa=\frac{e^{\lambda\left(t_{0}-t\right)}}{(1-c)^{2}}\left(\frac{d^{2}}{1-c}+d^{2} c+\frac{M_{\delta}}{c}\right)+\frac{2}{(1-c)^{2}} \int_{-\infty}^{t_{0}} e^{\lambda s} \beta(s) d s+\frac{M_{\delta} e^{\lambda t_{0}}}{c(1-c)} \\
\kappa^{\prime}=\frac{\varepsilon^{-1}}{(1-c)^{2}} \sum_{i=1}^{m} L_{i}^{2}
\end{gathered}
$$

and $\gamma=\lambda$. Then, taking into account that we have chosen $\varepsilon=\frac{\alpha_{0}}{m}$, it follows that

$$
\frac{\kappa^{\prime}}{\gamma}=\frac{\varepsilon^{-1}(1-c)^{-2} \sum_{i=1}^{m} L_{i}^{2}}{\lambda}<1
$$

Thus, Lemma 1 implies that

$$
\begin{aligned}
|x(\tau)|^{2} & \leq \kappa e^{-\mu \tau} \\
& =\left(\frac{e^{\lambda\left(t_{0}-t\right)}}{(1-c)^{2}}\left(\frac{d^{2}}{1-c}+d^{2} c+\frac{M_{\delta}}{c}\right)+\frac{2}{(1-c)^{2}} \int_{-\infty}^{t_{0}} e^{\lambda s} \beta(s) d s+\frac{M_{\delta} e^{\lambda t_{0}}}{c(1-c)}\right) e^{-\mu \tau},
\end{aligned}
$$

where $\mu$ is the solution of the next equation which belongs to the interval $(0, \lambda)$ :

$$
\frac{\kappa^{\prime}}{\lambda-\mu} e^{\mu r}=1
$$

Whence we obtain that

$$
\begin{aligned}
& \sup _{\theta \in[-r, 0]}\left|x\left(t_{0}+\theta\right)\right|^{2} \\
& \leq \frac{e^{\mu r}}{(1-c)^{2}}\left(\frac{d^{2}}{1-c}+d^{2} c+\frac{M_{\delta}}{c}\right) e^{(\lambda-\mu) t_{0}} e^{-\lambda t}+\frac{2 e^{\mu r}}{(1-c)^{2}} \int_{-\infty}^{t_{0}} e^{\lambda s} \beta(s) d s \\
& \quad+\frac{M_{\delta} e^{\lambda t_{0}}}{c(1-c)} e^{\mu r} \\
& \leq\left(1+\frac{M_{\delta} e^{\lambda t_{0}}}{c(1-c)}+\frac{2}{(1-c)^{2}} \int_{-\infty}^{t_{0}} e^{\lambda s} \beta(s) d s\right) e^{\mu r}
\end{aligned}
$$


provided

$$
t \geq T_{D}=\lambda^{-1} \log \left(\frac{1}{(1-c)^{2}}\left(\frac{d^{2}}{1-c}+d^{2} c+\frac{M_{\delta}}{c}\right) e^{(\lambda-\mu) t_{0}}\right) .
$$

Consequently, the family of bounded sets $\{B(t)\}_{t \in \mathbb{R}}$ in $C\left([-r, 0] ; \mathbb{R}^{n}\right)$ given by $B(t):=B(0 ; R(t))$, for all $t \in \mathbb{R}$, where $B(0 ; R(t))$ denotes the ball centred at zero with radius $R(t)=$ $\left(1+\frac{M_{\delta} e^{\lambda t}}{c(1-c)}+\frac{2}{(1-c)^{2}} \int_{-\infty}^{t} e^{\lambda s} \beta(s) d s\right) e^{\mu r}$, is absorbing. Taking into account again that the associated process maps bounded sets into bounded sets of $C\left([-r, 0] ; \mathbb{R}^{n}\right)$, the existence of the pullback attractor is ensured again by Theorem 2 .

\section{Conclusion}

In this paper we presented a big improvement of some previous results on the asymptotic behaviour of solutions to non-linear neutral (or non-neutral) delay differential equations recently published (see [Caraballo \& Kiss, 2013a,b]). Namely, our improvement states that if a non-autonomous differential system possesses a dissipative term without delay satisfying certain integrability condition (namely condition (5)), then for any suitable Lipschitz perturbation containing measurable variable delays, the perturbed system also possesses a pullback attractor.

\section{Acknowledgments}

We would like to thank the referees for their useful comments. The research of T.C. is partly supported by Ministerio de Economía y Competitividad (Spain) under grant MTM2011-22411. The work of G.K. is partially supported by the grant MTM2011-24766 of MICINN (Spain), as well as by the already mentioned MTM2011-22411. The investigation of T.T. is partially supported by Grant-in-Aid for Scientific Research (no. 24540198) of the Japan Society for the Promotion of Science.

\section{References}

Caraballo, T. \& Kiss, G. [2013a] "Attractivity for neutral functional differential equations," Discrete and Continuous Dynamical Systems - B 18, 1793-1804. doi:10.3934/dcdsb.2013.18.1793, URL http://dx. doi.org/10.3934/dcdsb.2013.18.1793.

Caraballo, T. \& Kiss, G. [2013b] "Attractors for differential equations with multiple variable delay," Discrete and Continuous Dynamical Systems-A 33, 1365-1374. doi:10.3934/dcds.2013.33.1365, URL http:// dx.doi.org/10.3934/dcds.2013.33.1365.

Caraballo, T., Real, J. \& Taniguchi, T. [2007] "The exponential stability of neutral stochastic delay partial differential equations," Discrete Contin. Dyn. Syst. 18, 295-313, doi:10.3934/dcds.2007.18.295, URL http://dx.doi.org/10.3934/dcds.2007.18.295.

Chen, H. [2010] "Impulsive-integral inequality and exponential stability for stochastic partial differential equations with delays," Statist. Probab. Lett. 80, 50-56, doi:10.1016/j.spl.2009.09.011, URL http: //dx.doi.org/10.1016/j.spl.2009.09.011.

Hale, J. K. \& Verduyn Lunel, S. M. [1993] Introduction to functional-differential equations, Applied Mathematical Sciences, Vol. 99 (Springer-Verlag, New York), ISBN 0-387-94076-6.

Mallet-Paret, J. \& Nussbaum, R. D. [2013] "Analyticity and nonanalyticity of solutions of delay-differential equations," arXiv preprint arXiv:1305.0579 .

Schmalfuss, B. [1992] "Backward cocycles and attractors of stochastic differential equations," International Seminar on Applied Mathematics-Nonlinear Dynamics: Attractor Approximation and Global Behaviour (Dresden), pp. 185-192. 